\title{
Techniques in Total Mesorectal Excision Surgery
}

\author{
Warren E. Lichliter, MD, FACS, FASCRS ${ }^{1}$ \\ ${ }^{1}$ Division of Colon and Rectal Surgery, Baylor University Medical \\ Center, Dallas, Texas \\ Clin Colon Rectal Surg 2015;28:21-27.
}

\begin{abstract}
Address for correspondence Warren E. Lichliter, MD, FACS, FASCRS, Division of Colon and Rectal Surgery, Baylor University Medical Center at Dallas, 1st Floor, Roberts Hospital, 3500 Gaston Avenue, Dallas, TX 75246 (e-mail: WARRENL@baylorhealth.edu).
\end{abstract}
Abstract
Keywords
- rectal cancer
- total mesorectal excision
- surgical technique

\begin{abstract}
Advances in the surgical management of rectal cancer have placed the quality of total mesorectal excision (TME) as the major predictor in overall survival. A standardized TME technique along with quality increases the percentage of patients undergoing a complete TME. Quality measurements of TME will place increasing demands on surgeons maintaining competence with present and future techniques. These efforts will improve the outcome of the rectal cancer patients.
\end{abstract}

It is helpful to understand the progression of concepts and techniques in the management of rectal surgery-from initial local resection of the tumor to radical and sphincter-saving procedures; from the concept of clear radial margin to the total mesorectal excision (TME). It is also important to give credit to the years of pioneering work it took to develop what is now the standard of care in rectal cancer surgery. Miles in 1908 , dissatisfied with the perineal approach to rectal cancer, reported on his experience on abdominoperineal resection (APR), creating the ability to resect rectal cancer with improved outcomes. In this article, we described Miles' three zones of lymphatic spread, the upward spread being the most important. ${ }^{1}$ The development of circular staplers in the 1970s led to sphincter-saving procedures and the standard distal margin of resection of $5 \mathrm{~cm} .{ }^{2,3}$ With increasing experience in stapling techniques, $2 \mathrm{~cm}$ became the accepted distal margin. ${ }^{4,5}$ This has further been defined in the setting of neoadjuvant therapy as a "negative" histologic margin. ${ }^{6-8}$ The concept of circumferential margin (CRM) was initially described by Quirke in 1976 (Prof. P. Quirke, PhD, personal communication). Subsequently, its importance as a predictor of local recurrence has been shown in numerous articles. ${ }^{9-11}$ Heald, in a series of articles, defined a complete TME. ${ }^{12-14} \mathrm{~A}$ landmark publication by Quirke in 1986 along with Heald's work launched an evolving concept of TME and CRM into the central component of what is now the standard of care in rectal cancer surgery. ${ }^{15}$ Currently, the Consortium for Optimizing Surgical Treatment of Rectal Cancer (OSTRiCh) is working to develop a process that incorporates these concepts into defining what will become centers of excellence in the surgical management of rectal cancer. ${ }^{16}$

Issue Theme Rectal Cancer; Guest Editors: James W. Fleshman, MD, FACS, FASCRS, and Warren E. Lichliter, MD, FACS, FASCRS

\section{Total Mesorectal Excision as a Measure of Quality}

As TME has emerged as a standard of care, the measurement of the "quality" of the TME specimen is helping to define the surgical quality of both the surgeon and institution. The same authors that helped define TME have also shown that an incomplete TME is associated with an increased risk of recurrence and a decreased overall survival. $^{9-11,14-17}$ The "completeness" of TME defined as complete, near complete, and inadequate, and institutional outcomes reporting has been proposed with work in Europe led by Drs. Quirke and Heald from 1976 to 1986 . $^{11,14,15}$ More recent European data as well as increasing reports from the United States have supported these findings. ${ }^{17-19}$ All of these efforts have emphasized the importance of standardizing techniques in surgery and pathology for rectal cancer. Measuring quality outcomes provides feedback and incentive to achieve a high rate of complete TME and decreased local recurrence rates. Most of these studies are related to open surgery. Multiple studies including the COLOR and CLASSIC trials have now shown equivalent outcomes in laparoscopic or open technique. ${ }^{20-23}$ Although outcomes of SILS lap or robotic, hybrid robotic, or robotic surgical techniques are early, outcomes seem to be equivalent to open procedures. ${ }^{24-26}$ All of these developing techniques in surgery will be held to standards of the quality of the surgical dissection. Currently, the quality of TME surgery in rectal cancer and the number of lymph nodes in colon cancer are the only quality metrics in oncologic surgery.
Copyright (c) 2015 by Thieme Medical Publishers, Inc., 333 Seventh Avenue, New York, NY 10001, USA. Tel: +1(212) 584-4662.
DOI http://dx.doi.org/ 10.1055/s-0035-1545066. ISSN 1531-0043. 


\section{Defining Total Mesorectal Excision}

A complete understanding of the definition of a complete TME is needed in trying to describe surgical techniques in achieving a complete TME. A complete TME has been defined as a "complete removal of the lymph node bearing mesorectum along with its intact enveloping fascia." ${ }^{27}$ The complete TME has components including (1) high ligation of the inferior mesenteric artery (IMA), (2) complete mobilization of the splenic flexure, (3) division of the colon at the descending sigmoid junction, (4) sharp dissection in the avascular plane into the pelvis anterior the presacral fascia and outside the fascia propria or enveloping visceral fascia, (5) division of lymphatic and middle hemorrhoidal vessels anterolaterally, and (6) inclusion of all pelvic fat and lymphatic material at least $2 \mathrm{~cm}$ below the level of the distal margin. All of these criteria have been validated by multiple studies and are the framework for current practice guidelines. ${ }^{8,28} \mathrm{~A}$ complete TME will not be met if these basic components are not met at surgery. An incomplete resection is often encountered with violation of the posterior rectum in a difficult pelvis or low anastomosis when the muscle is exposed only to find that the tumor margin needs to be lower. Difficulty in defining the rectovaginal septum or posterior wall of the prostate and/or seminal vesicles also results in the entry into the anterior wall of the rectum and an incomplete TME.

\section{Measuring Quality}

\section{Operative Assessment of Total Mesorectal Excision}

The first three components of a complete TME are related to intraoperative decision making with the ligation of the IMA, mobilization of the splenic flexure, and resection of the entire sigmoid colon. None of these components are evaluated by the pathologist, so it is up to the surgeon to be diligent in performing these components and to document these steps in its operative dictation. ${ }^{8} \mathrm{~A}$ lower ligation of the IMA, only partial mobilization of the splenic flexure, or the use of the sigmoid colon may be appropriate in selected patients but the reason for these decisions should be documented in the operative note. Although there may be no significant oncologic advantage to a high ligation of the IMA, this may facilitate a more complete mobilization of the left colon (especially with a medial to lateral technique) to better ensure adequate blood supply and a tension-free anastomosis. ${ }^{29}$ Defining the plane between the inferior mesenteric artery (IMV) and IMA with a high ligation can also help in the identification of the left ureter. ${ }^{30,31} \mathrm{~A}$ complete mobilization of the left colon may not be needed in all cases but reports of anastomotic leaks have been related to failure to completely mobilize the flexure. ${ }^{32}$ This is especially the case in the obese patient who often has a foreshortened mesentery. ${ }^{33}$ Dividing the colon at the junction of the left colon and sigmoid colon at a right angle to the mesentery facilitates a complete lymphatic dissection and entry into the retroperitoneum at the sacral promontory. The use of the sigmoid colon has been associated with a higher risk of anastomotic complications. ${ }^{34}$

\section{Grading the Total Mesorectal Excision Specimen}

A complete discussion on the processing of the TME specimen by the pathologist is presented in the next chapter. Standardizing the processing and reporting of the macroscopic quality of the TME and the microscopic CRM specimen has been led by Quirke, Heald, and Nagtegaal. ${ }^{35-37}$ Several aspects of the surgeon's handling of the specimen are important. Orientating the specimen with the pathologist in the operating room is ideal. The TME specimen should be received by pathology as an intact and unopened specimen to allow for proper handling and assessment by the pathologist. Only if a distal margin needs to be assessed should the specimen be opened and this should be documented and ideally done with the pathologist present. Gross photos of the intact specimen may need to be performed by the surgeon and with the ubiquitous presence of "smart phones" this should be easily done. The specimen should be oriented with the proximal end to the left and the distal end toward the right. A ruler should be placed in the picture for scale. Photos of the anterior and posterior specimen are taken. Photos of any potential defects should be taken if present.

Grading the mesorectum is defined by the College of American Pathologists (CAP) as complete, nearly complete, and incomplete. A complete TME, or mesorectal plane, has an intact mesorectum with only minor irregularities of less than $5 \mathrm{~mm}$. There is no coning toward the distal margin, and a smooth CRM on transverse sections of the mesorectum. A nearly complete TME, or intramesorectal plane, has one or more defects in the mesorectum greater than $5 \mathrm{~mm}$ into the mesorectum, moderate coning with no visible muscularis propria, and irregular CRM on transverse sectioning. An incomplete, or muscularis propria plane, has exposed muscularis propria, moderate to marked coning, and irregular CRM on transverse sections. ${ }^{38}$ The quality of the TME is determined not only with photography and analysis of the gross specimen but also with the cross-sectional macroscopic photos of the TME. ${ }^{39}$

The sphincter complex in an APR is graded as extralevator, sphincter plane, and intrasphincteric. An extralevator specimen has a cylindrical specimen with no waist effect and the levators are removed en bloc. A sphincteric plane has a slight waist effect and no significant defects or perforations. An intrasphincteric/submucosal plane has a significant waist effect with perforation or missing areas of muscularis propria.

After the specimen has been photographed and fixed in formalin, the TME is cross-sectioned at 3 to $5 \mathrm{~mm}$ intervals and prepared for photography. The top left of the photograph should be the most proximal transverse section and the bottom right for the most distal section. They should also orient the cross-sections to correlate with MRI imaging. Left is viewer's right, right is viewer's left. Defects in the inked margin on cross-sections may change the TME grading. A positive margin on CRM is less than $1 \mathrm{~mm}$ distance from the primary tumor, tumor extension, positive lymph node, or tumor deposit. Acellular pools of mucin at the level of the CRM after neoadjuvant radiochemotherapy are considered negative.

The highest quality measures of the surgical procedure for a rectal cancer (circumferential radial margin, distal margin, 
and TME) are the focus on the just completed, unpublished ACOSOG Z6051 trial comparing hand-assisted laparoscopy and open surgery for rectal cancer. In practice, the ideal outcome being measured today should include the accurate reporting and dictation by the surgeon concerning all six components of a complete TME and a pathologic evaluation and reporting of a CRM of less than $1 \mathrm{~mm}$, a negative distal margin, and a complete TME. ${ }^{40}$

\section{Surgical Techniques for Total Mesorectal Excision}

\section{General Concepts for Total Mesorectal Excision}

In both open procedures and minimally invasive procedures, oncologic principles always apply. This is difficult surgery made more complicated by the increasing age, weight, and frailty of the rectal cancer patient. Rapid advances in the technical aspect of surgery have resulted in the demands placed on surgeons to become competent with techniques that have (by necessity) been learned postresidency. Although instrumentation such as staplers, thermal devices, laparoscopy, and robotics has made the minimally invasive operation possible, the surgeon is still faced with limited exposure, altered anatomy, and low pelvic dissections with less than optimal limited instrumentation for the sphinctersaving procedure or APR. Even newer techniques with transabdominal transanal, retrograde TME, etc., push these boundaries. It is very important to remember that safe surgery is still the priority with a patient's care. A realistic appraisal of the patient and surgeon's level of expertise preoperatively can help avoid intraoperative injuries and prolonged surgery. ${ }^{41,42}$ It has been shown that conversion from laparoscopic or robotic to a hand-assisted procedure can avoid conversion to an open procedure. ${ }^{43}$ It has also been shown recently that beyond 3 to 4 hours of a minimally invasive approach outcomes are not improved over an open procedure. ${ }^{44}$ A surgeon must not let new technology get in the way of optimizing the safety and ultimate well-being of the patient. A conversion to an open procedure may be a wise decision and not a failure of technique. The conversion to open may still result in a difficult procedure but the exposure and other factors make it a safer procedure.

Most of the initial literature from Europe relative to a TME dissection looked at open procedures. Because of this newer reports such as the COST, Classic, JCOG 0404, or the recent ACOSOG Z6051, all have been designed to show equivalency or noninferiority to open techniques. ${ }^{21,22,24,45}$ A randomized study has also shown equivalency in hand-assisted laparoscopy versus laparoscopic colorectal surgery. ${ }^{46}$ All of these trials included only surgeons qualified in both procedures. All of these studies showed similar oncologic outcomes with similar rates of morbidity and mortality, oncologic outcomes, and lymph node yields. The classic trial, as well as others, has shown an increased risk of complications with a laparoscopic approach relative to bowel injury and ureteral injury. In addition, an increased rate of conversion has been shown for surgery in the obese. All of these studies outline the potential for poor outcomes if good judgment is not utilized.

\section{Open Total Mesorectal Excision}

Several factors are important before the procedure has begun: lithotomy position with careful leg positioning, and placement of the buttocks low enough on the OR table to advance an EEA instrument for an unexpected higher anastomosis. A bowel prep, either oral or with enemas to facilitate rectal anastomosis, is important. Although not the scope of this chapter, the use of an oral antibiotic is being reexamined.

The initial aspects of the TME are related to the ligation of the IMA, mobilization of the splenic flexure, and division of the left colon and sigmoid junction. The initial procedure after exploration is mobilization along the left gutter and the white line of Toldt. The splenic flexure should be completely mobilized from its abdominal wall and peritoneal attachments. A decision on the division of the mesentery and blood supply can be deferred until the length of colon needed can be better defined. As with laparoscopy, avoid dissections too wide along the gutter and reflecting the kidney. Dissection high in the mesentery at the level of the splenic flexure can injure the arcade vessel along the colon. Watch for the ureter medially and toward the renal pelvis. Just lateral to the ligament of Treitz is the IMV. Once the flexure is mobilized, the lateral to medial dissection can begin down the left pelvic sidewall to identify the ureter over the anterior surface of the common iliac artery and the IMA on the anterior aorta.

\section{Posterior Plane}

The posterior plane and the identification of the IMA are integral features of the TME. Most descriptions of an open technique describe a lateral or conventional approach to the posterior plane, with identification of the IMA and ureter. With the advent of laparoscopic techniques, a medial to lateral approach has been advocated. Being proficient in utilizing both techniques would be wise. If there is confusing anatomy, opening up the lateral peritoneum and working well below the pelvic brim and dissecting back up the pelvic inlet can be useful in defining the IMA and the ureter. Once the IMA is divided, the entrance into the Holy Plane is established. Avoid blunt dissection. Work initially in the presacral plane anterior to the endopelvic fascia and outside the mesorectal membrane. Working in the presacral plane from medial to lateral when in the posterior plane helps define the lateral pelvic sidewall with ureter and iliac veins. As the dissection proceeds inferiorly, retracting the rectum and mesorectum from side to side helps to expose the lateral borders of the dissection. Working from posterior to anterior helps avoid coning in to the rectal wall and reduces the risk of positive radial margins. As the dissection reaches the pelvic floor, work from posterior to anterior to stay away from the sacral nerve branches. A St Mark's pelvic retractor with a lip is valuable in both areas of dissection. The lateral dissection in the deep pelvis can be facilitated by an energy device. In a narrow pelvis, a ribbon retractor can help to supply the retraction needed when moving the mesorectum to the right or left to expose the right or left gutter. 


\section{Ureter Identification}

The ureter is usually identified from a lateral to medial approach but the medial to lateral may be needed depending on anatomy or tumor characteristics. The ureter can be injured in three locations, usually at the pelvic brim but also more distally along the deep sidewall and again at the base of the bladder. Trying to achieve a high ligation without utilizing dissection in known embryologic planes can injure the ureter above the pelvic brim. Avoid coning in on the rectum in a low pelvic dissection but be aware that the dissection anteriorly gets closer to base of bladder and the trigone. This can be more of a problem in a female patient with prior hysterectomy and altered anatomy at the base of the bladder and the vaginal cuff. Placement of ureteral stents can be helpful. A recent review of ureteral injuries showed that stents did not prevent injury and not all injuries were recognized intraoperatively. ${ }^{47}$

\section{Nerve Preservation}

The main sacral nerves lie superficially in the plane between the peritoneum and the endopelvic fascia at the level of the pelvic brim or just below. The sacral plexus in the pelvic floor is diffused and located at the pelvic floor posterior and lateral to the mesorectum. These are the two areas where injury is more likely. Do not compromise a complete TME to dissect the nerves from the posterior fascia propria. Sacrifice only the nerves entering into the mesorectum that are well displayed crossing the areolar plane posterior to the fascia of the mesorectum. Though little data reflect potential injury from energy devices or electrocautery, sharp division of the nerve could be considered.

\section{Vasculature}

Identification of the IMA has been described with either a lateral to medial or medial to lateral dissection. Traction on the sigmoid colon superiorly with division of the right lateral pelvic surface to expose the areolar plane outside the mesorectum is helpful. The IMA is usually doubly ligated with an open technique but thermal devices can be appropriate. Be aware of tortuous or aneurysmal iliac arteries as this can confuse ureteral anatomy. With wider dissections and more aggressive management of larger lesions, the iliac vessels, especially the vein, can be injured. Adequate blood supply of the proximal bowel is increasingly important for successful low anastomosis in the deep pelvis (coloanal), and it may be necessary to resect up to the level of the left colon to reach vascularized tissue adequate for anastomosis. SPY Technology has been used along with more traditional measures such as the "flash test." A recent review of the indocyanine green evaluation of anastomatic perfusion experience failed to demonstrate any outcome improvement but may help with decision making intraoperatively. ${ }^{48}$

\section{Pelvic Floor and Distal Margin}

Once the posterior dissection proceeds to a level distal to the tumor, a tumor-specific TME can be done if the lesion is in the upper rectum or distal sigmoid colon. For mid to low rectal cancers, the posterior dissection should continue to the pelvic floor for a complete resection of the mesorectum. Division of Waldeyer fascia defines the inferior aspect of the mesorectum. The remaining adipose tissue should be divided at the level or just below the distal margin. Divide the posterior tissues at the distal margin at right angles. Avoid, if possible, dividing the mesorectum and then finding an inadequate distal margin. The exposed muscle of the rectum above the distal margin results in an incomplete TME. To identify the anterior plane of dissection in the deep pelvis, the St Mark's retractor is valuable. The vaginal wall and rectovaginal septum can be better identified, especially after a previous hysterectomy, with an EEA sizer in the vaginal vault reflected anteriorly. Once the distal margin has been dissected out, conventional staplers and stapling techniques are used. On occasion, the pelvis is too narrow for stapler access. The entire specimen can be delivered through the rectum and a stapling instrument can then be fired in the perineum just below the prolapsed dentate line. Bringing the specimen through the rectum can be facilitated by using the EEA and umbilical tape to prolapse the bowel through the rectum.

\section{Laparoscopic Total Mesorectal Excision}

The same general operative principles of an open procedure apply to laparoscopic or minimally invasive procedures. Lithotomy position is important. With the increased need for head down, head up, and lateral rotation, consideration should be given for fixation of the patient on a foam pads or a bean bag to prevent patient slipping on the OR table. ${ }^{49}$ The right arm is usually tucked in to allow positioning for the surgeon and camera holder. A bowel prep facilitates management of the rectal anastomosis. The splenic flexure is sometimes easier performed with a medial to lateral dissection with division of the IMA first. This opens up the dissection proceeding superiorly along the base of left colon mesentery to the IMV and splenic flexure.

\section{Posterior Plane}

The posterior plane is usually identified and entered after the division of the IMA. With a pneumoperitoneum, the initial division of the gutter of the right mesorectum inferior to the IMA at the pelvic brim can open up the posterior plane anterior to the presacral fascia to facilitate identification of structures at the pelvic brim. The remainder of the posterior dissection is the same as described with an open TME. To obtain exposure with a large uterus, a monofilament suture may be needed to place through the abdominal wall to suture the uterus anteriorly.

\section{Ureteral Identification}

Identification of the ureter can be facilitated by the magnification of the camera and close visualization of the sidewall. A recent review and meta-analysis of the medial to lateral approach to ureteral identification found shorter operative time, possible less rate of conversion, and questionably less blood loss. ${ }^{50} \mathrm{~A}$ lateral approach may be needed in a patient with a large pelvic mass or altered anatomy from inflammation or in any patient where a medial to lateral approach is not working. The ureter can be located between the IMV and IMA, 
at which point it is lateral to the gonadal vessels. It can be traced distally into the pelvis. Retracting the IMA anteriorly and opening up the dorsal plane below the IMA is important with the medial to lateral approach. Lighted ureteral catheters are helpful if available. Nonlighted stents are easily palpated if a hand-assisted technique is used. The same locations for injury apply to both open and laparoscopic techniques. A recent review of ureteral injuries did suggest a higher incidence of injury with laparoscopy. ${ }^{51}$ Importantly, even stents did not prevent injury and not all injuries were identified even when a stent was divided. ${ }^{47}$

\section{Nerve Preservation}

Laparoscopic techniques with a magnified camera have been shown to be better able to identify the sacral nerves not only at the pelvic brim but also in the deep lateral pelvic floor. Nerve preservation is accomplished with the same technique described for an open technique. There are conflicting reports on preservation of sexual function. Although visualization may be improved with magnification by the camera at the sacral promontory, randomized trials have shown increased nerve dysfunction with laparoscopy compared with open procedures.

\section{Vasculature}

Identification of the IMA may be easier especially as experience is gained with the medial to lateral approach. The main risk with the IMA may be increasing reliance on the use of energy devices and postoperative bleeding. Injury to the arcade vessel in the left colon can occur if the bowel is not retracted properly, and this injury can jeopardize the blood supply of the proximal bowel. The pelvic sidewall and iliac vein may also be prone to injury just as in open procedures. If the blood supply of the proximal bowel is questionable, newer techniques using indocyamine green dye (SPY, Firefly) can be used in a hand assisted laparoscopy or robotic case.

\section{Pelvic Floor and Distal Margin}

The same techniques in the deep pelvic floor apply to laparoscopic and open procedures. The uterus may need to be retracted with a suture to the abdominal wall. An EEA sizer in the vagina retracted superiorly can facilitate the identification of the posterior vaginal wall and separate it from the anterior rectal wall. Dividing Waldeyer fascia defines the inferior extent of the mesorectum. As with open techniques, avoid entering the posterior plane above the eventual distal margin resulting in an incomplete TME. Avoid coning in on the levator floor. This is especially a problem with APR resection of a low cancer, again resulting in an incomplete TME.

\section{Managing the Difficult Pelvis}

The difficult pelvis is a problem regardless of operative techniques contemplated by the surgeon. The obese patient, male patient, and patients with prior surgery are all at increased risk for intraoperative injuries, incomplete TME, positive CRM, and failed anastomosis. ${ }^{52}$ Several factors are important. The increased adipose tissue reduces peritoneal space and diminishes the "doming effect" of pneumoperitoneum. Foreshortening of the mesentery makes it harder to retract the small bowel. It also makes it difficult to identify vasculature at the IMA and sidewall of pelvis. The foreshortened colonic mesentery makes a complete mobilization of the spleen flexure mandatory to allow adequate length for a low anastomosis. The difficulty in exposure places the patient at increased risk for positioning injury if extremes of body positioning are utilized. The increased fat in the pelvis along with a narrow pelvis can make retraction for exposure challenging. All of these factors explain why numerous reports on laparoscopy in the obese is associated with higher conversion rates, increased operative time, and increased morbidity and mortality. ${ }^{53,54}$ These factors are also why initial trials of laparoscopy excluded the morbidly obese patient. The following discussion reviews steps to manage the difficult pelvis.

In open cases, attempting to operate through a small incision leads to inability to identify structures in a pelvis distorted by encroachment of fat and loss of abdominal domain. A larger incision can provide more adequate exposure. Division of the colon and packing the proximal end of the colon in the upper abdomen with the small bowel can help expose the pelvis at the pelvic brim. The use of a "lipped" St Mark's retractor is also useful. The use of ureteral stents may help with identification of both the right and left ureter. The use of an EEA sizer in the vaginal vault can help identify the rectovaginal septum during the anterior mesorectal dissection. ${ }^{55}$

In laparoscopic cases, a preoperative assessment can help to decide whether a hand-assisted laparoscopy would be more appropriate. The decision could also be made whether an open procedure is more appropriate following a laparoscopic mobilization of the splenic flexure. This could be important especially in a large patient with a large or lowlying tumor. At the time of the procedure, an extra port may be useful to provide better exposure. As in open procedures, ureteral stents can be helpful. Lighted stents will be needed for laparoscopic procedures but nonlighted stents are adequate for a hand-assisted procedure. The bulky visceral fat makes identification of structures from a medial to lateral approach difficult. A lateral to medial or inferior to superior approach may be needed.

The role of a hand-assisted laparoscopic procedure overcomes some of the limitations of conventional laparoscopic surgery. Multiple studies have confirmed equivalent outcomes to laparoscopy with shorter operative times and reduced conversion rates to open. A strong consideration should be given in converting a laparoscopic case to a hand assist before converting to open.

\section{Summary}

Recent advances in the development of the surgical management of rectal cancer have placed the quality of TME as the major predictor in overall survival of the rectal cancer patient. Knowledge of the definition of a quality TME and the increasing standardization of a TME dissection utilizing techniques 
discussed in this chapter will facilitate the increasing percentage of patients undergoing a complete TME. The ability to measure the quality of a TME will continue to place increasing demands on surgeons maintaining competence not only with present techniques but also in the many advances to come. With all these efforts, the outcome of the rectal cancer patient will surely improve.

\section{References}

1 Miles WE. A method of performing abdomino-perineal excision for carcinoma of the rectum and of the terminal portion of the pelvic colon (1908). CA Cancer J Clin 1971;21(6):361-364

2 Dixon CF. Anterior resection for malignant lesions of the upper part of the rectum and lower part of the sigmoid. Ann Surg 1948; 128(3):425-442

3 Goligher JC, Dukes CE, Bussey HJ. Local recurrences after sphincter saving excisions for carcinoma of the rectum and rectosigmoid. $\mathrm{Br}$ J Surg 1951;39(155):199-211

4 Fain SN, Patin CS, Morgenstern L. Use of a mechanical suturing apparatus in low colorectal anastomosis. Arch Surg 1975;110(9): 1079-1082

5 Pollett WG, Nicholls RJ. The relationship between the extent of distal clearance and survival and local recurrence rates after curative anterior resection for carcinoma of the rectum. Ann Surg 1983;198(2):159-163

6 Kuvshinoff B, Maghfoor I, Miedema B, et al. Distal margin requirements after preoperative chemoradiotherapy for distal rectal carcinomas: are $<$ or $=1 \mathrm{~cm}$ distal margins sufficient? Ann Surg Oncol 2001;8(2):163-169

7 Park IJ, Kim JC. Adequate length of the distal resection margin in rectal cancer: from the oncological point of view. J Gastrointest Surg 2010;14(8):1331-1337

8 Tjandra JJ, Kilkenny JW, Buie WD, et al; Standards Practice Task Force; American Society of Colon and Rectal Surgeons. Practice parameters for the management of rectal cancer (revised). Dis Colon Rectum 2005;48(3):411-423

9 Quirke P. Training and quality assurance for rectal cancer: 20 years of data is enough. Lancet Oncol 2003;4(11):695-702

10 Quirke P, Durdey P, Dixon MF, Williams NS. Local recurrence of rectal adenocarcinoma due to inadequate surgical resection. Histopathological study of lateral tumour spread and surgical excision. Lancet 1986;2(8514):996-999

11 Adam IJ, Mohamdee MO, Martin IG, et al. Role of circumferential margin involvement in the local recurrence of rectal cancer. Lancet 1994;344(8924):707-711

12 Heald RJ. A new approach to rectal cancer. Br J Hosp Med 1979; 22(3):277-281

13 Heald RJ. The 'Holy Plane' of rectal surgery. J R Soc Med 1988; 81(9):503-508

14 Heald RJ, Husband EM, Ryall RD. The mesorectum in rectal cancer surgery-the clue to pelvic recurrence? Br J Surg 1982;69(10): 613-616

15 Morris E, Quirke P, Thomas JD, Fairley L, Cottier B, Forman D. Unacceptable variation in abdominoperineal excision rates for rectal cancer: time to intervene? Gut 2008;57(12):1690-1697

16 Abbas MA, Chang GJ, Read TE, et al; Consortium for Optimizing Surgical Treatment of Rectal Cancer. Optimizing rectal cancer management: analysis of current evidence. Dis Colon Rectum 2014;57(2):252-259

17 Scott N, Jackson P, al-Jaberi T, Dixon MF, Quirke P, Finan PJ. Total mesorectal excision and local recurrence: a study of tumour spread in the mesorectum distal to rectal cancer. Br J Surg 1995; 82(8):1031-1033
18 Maurer CA, Renzulli P, Kull C, et al. The impact of the introduction of total mesorectal excision on local recurrence rate and survival in rectal cancer: long-term results. Ann Surg Oncol 2011;18(7): 1899-1906

19 Martling AL, Holm T, Rutqvist LE, Moran BJ, Heald RJ, Cedemark B. Effect of a surgical training programme on outcome of rectal cancer in the County of Stockholm. Stockholm Colorectal Cancer Study Group, Basingstoke Bowel Cancer Research Project. Lancet 2000;356(9224):93-96

20 Hainsworth PJ, Egan MJ, Cunliffe WJ. Evaluation of a policy of total mesorectal excision for rectal and rectosigmoid cancers. Br J Surg 1997;84(5):652-656

21 Buunen M, Bonjer HJ, Hop WC, et al; Color II Study Group. COLOR II. A randomized clinical trial comparing laparoscopic and open surgery for rectal cancer. Dan Med Bull 2009;56(2):89-91

22 Kitano S, Inomata M, Sato A, Yoshimura K, Moriya Y; Japan Clinical Oncology Group Study. Randomized controlled trial to evaluate laparoscopic surgery for colorectal cancer: Japan Clinical Oncology Group Study JCOG 0404. Jpn J Clin Oncol 2005;35(8):475-477

23 Guillou PJ, Quirke P, Thorpe H, et al. MRC CLASSIC trial group. Short-term endpoints of conventional versus laparoscopic-assisted surgery in patients with colorectal cancer (MRC CLASSIC trial): multicentre, randomised, controlled trial. Lancet 2005; 365:1718-1726

24 Clinical Outcomes of Surgical Therapy Study Group. A comparison of laparoscopically assisted and open colectomy for colon cancer. $\mathrm{N}$ Engl J Med 2004;350(20):2050-2059

25 Baek JH, McKenzie S, Garcia-Aguilar J, Pigazzi A. Oncologic outcomes of robotic-assisted total mesorectal excision for the treatment of rectal cancer. Ann Surg 2010;251(5):882-886

26 deSouza AL, Prasad LM, Ricci J, et al. A comparison of open and robotic total mesorectal excision for rectal adenocarcinoma. Dis Colon Rectum 2011;54(3):275-282

27 Trastulli S, Farinella E, Cirocchi R, et al. Robotic resection compared with laparoscopic rectal resection for cancer: systematic review and meta-analysis of short-term outcome. Colorectal Dis 2012; 14(4):e134-e156

28 Nogueras JJ. Open low anterior resection. In: Wexner SD, Fleshman JW, Fischer JE, eds. Master Techniques in General Surgery: Colon and Rectal Surgery: Abdominal Operations. Philadelphia, PA: Lippincott Williams \& Wilkins; 2012:127-131

29 Monson JR, Weiser MR, Buie WD, et al; Standards Practice Task Force of the American Society of Colon and Rectal Surgeons. Practice parameters for the management of rectal cancer (revised). Dis Colon Rectum 2013;56(5):535-550

30 Lange MM, Buunen M, van de Velde CJH, Lange JF. Level of arterial ligation in rectal cancer surgery: low tie preferred over high tie. A review. Dis Colon Rectum 2008;51(7):1139-1145

31 Shin J, Lee SW. Laparoscopic complications. In: Steele SR, Maykel JA, Champagne BJ, Orangio GR, eds. Complexities in Colorectal Surgery. New York: Springer; 2014

32 Hiranyakas A, Da Silva G, Denoya P, Shawki S, Wexner SD. Colorectal anastomotic stricture: is it associated with inadequate colonic mobilization? Tech Coloproctol 2013;17(4):371-375

33 Efron JE, Vargas HD. The morbidly obese patient. In: Steele SR, Maykel JA, Champagne BJ, Orangio GR, eds. Complexities in Colorectal Surgery. New York: Springer; 2014

34 Karanjia ND, Corder AP, Bearn P, Heald RJ. Leakage from stapled low anastomosis after total mesorectal excision for carcinoma of the rectum. Br J Surg 1994;81(8):1224-1226

35 Quirke P, Steele R, Monson J, et al. MRC CR07/NCIC-CTG CO16 Trial Investigators; NCRI Colorectal Cancer Study Group. Effect of the plane of surgery achieved on local recurrence in patients with operable cancer: a prospective study using data from the MRC CR07 and NCIC-CTG CO16 randomised clinical trial. Lancet 2009; 373:821-828 
36 Quirke P, Dixon MF. The prediction of local recurrence in rectal adenocarcinoma by histopathological examination. Int $\mathrm{J}$ Colorectal Dis 1988;3(2):127-131

37 Nagtegaal ID, van de Velde CJ, van der Worp E, Kapiteijn E, Quirke P, van Krieken JH; Cooperative Clinical Investigators of the Dutch Colorectal Cancer Group. Macroscopic evaluation of rectal cancer resection specimen: clinical significance of the pathologist in quality control. J Clin Oncol 2002;20(7):1729-1734

38 Nagtegaal ID, Quirke P. What is the role for the circumferential margin in the modern treatment of rectal cancer? J Clin Oncol 2008;26(2):303-312

39 Jass JR, O'Brien MJ, Riddell RH, Snover DC; Association of Directors of Anatomic and Surgical Pathology. Recommendations for the reporting of surgically resected specimens of colorectal carcinoma. Hum Pathol 2007;38(4):537-545

40 Fleshman J. American College of Surgeons Oncology Group (ACOSOG)-Z6051. A Phase III prospective randomized trial comparing laparoscopic-assisted resection versus open resection for rectal cancer. Available at: http://clinicaltrials.gov/ct2/show/NCT00726622. Accessed August 17, 2009

41 Damle RN, Macomber CW, Flahive JM, et al. Surgeon volume and elective resection for colon cancer: an analysis of outcomes and use of laparoscopy. J Am Coll Surg 2014;218(6):1223-1230

42 Sammour T, Kahokehr A, Srinivasa S, Bissett IP, Hill AG. Laparoscopic colorectal surgery is associated with a higher intraoperative complication rate than open surgery. Ann Surg 2011;253(1): 35-43

43 Heneghan HM, Martin ST, Kiran RP, et al. Laparoscopic colorectal surgery for obese patients: decreased conversions with the handassisted technique. J Gastrointest Surg 2013;17(3):548-554

44 Bailey MB, Davenport DL, Vargas HD, Evers BM, McKenzie SP. Longer operative time: deterioration of clinical outcomes of laparoscopic colectomy versus open colectomy. Dis Colon Rectum 2014;57(5):616-622

45 Jayne DG, Guillou PJ, Thorpe H, et al; UK MRC CLASICC Trial Group. Randomized trial of laparoscopic-assisted resection of colorectal carcinoma: 3-year results of the UK MRC CLASICC Trial Group. J Clin Oncol 2007;25(21):3061-3068
46 Marcello PW, Fleshman JW, Milsom JW, et al. Hand-assisted laparoscopic vs. laparoscopic colorectal surgery: a multicenter, prospective, randomized trial. Dis Colon Rectum 2008;51(6): 818-826, discussion 826-828

47 Palaniappa NC, Telem DA, Ranasinghe NE, Divino CM. Incidence of iatrogenic ureteral injury after laparoscopic colectomy. Arch Surg 2012;147(3):267-271

48 Jafari MD, Wexner SD, Martz JE, McLemore EC, Margolin DA, Sherwinter DA, Lee SW, Senagore AJ, Phelan MJ, Stamos MJ. Perfusion Assessment in Laparoscopic left-sided/anterior resection (PILLAR II): a multi-institutional study. J Am Coll Surg 2015; 220(1):82-92.e1

49 Fleshman JW. Laparoscopic low anterior resection. In: Fleshman JW, Birbaum EH, Hunt SR, et al., eds. Atlas of Surgical Techniques for the Colon, Rectum, and Anus. Philadelphia, PA: Elsevier Saunders; 2013

50 Ding J, Liao GQ Xia Y, et al. Medial versus lateral approach in laparoscopic colorectal resection: a systematic review and metaanalysis. World J Surg 2013;37(4):863-872

51 Halabi WJ, Jafari MD, Nguyen VQ et al. Ureteral injuries in colorectal surgery: an analysis of trends, outcomes, and risk factors over a 10-year period in the United States. Dis Colon Rectum 2014;57(2):179-186

52 Delaney CP, Pokala N, Senagore AJ, et al. Is laparoscopic colectomy applicable to patients with body mass index $>30$ ? A case-matched comparative study with open colectomy. Dis Colon Rectum 2005; 48(5):975-981

53 Leroy J, Ananian P, Rubino F, Claudon B, Mutter D, Marescaux J. The impact of obesity on technical feasibility and postoperative outcomes of laparoscopic left colectomy. Ann Surg 2005;241: 69-762013

54 MakinoT, Shukla PJ, Rubino F, Milsom JW. The impact of obesity on perioperative outcomes after laparoscopic colorectal resection. Ann Surg 2012;255(2):228-236

55 Lin AY. Open low anterior resection of rectum. In: Fleshman JW, Birbaum EH, Hunt SR, et al., eds. Atlas of Surgical Techniques for the Colon, Rectum, and Anus. Philadelphia PA: Elsevier Saunders; 2013 